Original Paper

\title{
Pretreatment Neutrophil-Lymphocyte Ratio as a Predictor in Bladder Cancer and Metastatic or Unresectable Urothelial Carcinoma Patients: a Pooled Analysis of Comparative Studies
}

\author{
Shuiqing Wua,b Xiaokun Zhao $\quad$ Yinhuai Wang ${ }^{a} \quad$ Zhaohui Zhong ${ }^{a} \quad$ Lei Zhang $^{a}$ \\ Jian $\mathrm{CaO}^{\mathrm{a}}$ Kai $\mathrm{Ai}^{\mathrm{a}}$ Ran $\mathrm{Xu}^{\mathrm{a}}$ \\ aDepartment of Urology, The Second Xiangya Hospital, Central South University, Hunan Province, \\ People's Republic of China, ' $\mathrm{MRC}$ Centre for Reproductive Health, Queen's Medical Research Institute, \\ Edinburgh, United Kingdom
}

\section{Key Words}

Bladder cancer - Metastatic unresectable urothelial carcinoma - Unresectable urothelial carcinoma $\cdot$ Neutrophil-Lymphocyte ratio $•$ Prognosis

\begin{abstract}
Background/Aims: Emerging studies have shown that the neutrophil-lymphocyte ratio (NLR) is a potential predictor in various tumors. Our study was conducted to assess the prognostic value of the pretreatment NLR in bladder cancer and metastatic or unresectable urothelial carcinoma (mUC or UUC) patients up to July 2017. The correlation between the pretreatment NLR and pathological characteristics was also evaluated in bladder cancer patients. Methods: The hazard ratio (HR) and odds ratio (OR) with the $95 \%$ confidence interval $(\mathrm{CI})$ were extracted or calculated from the included studies for further pooled analysis. A total of 21 studies were included in a pooled analysis. Results: The pooled results indicated that a high pretreatment NLR was associated with reduced overall survival (OS) (HR=1.27, 95\% CI=1.12-1.43), relapsefree survival (RFS) $(H R=1.41,95 \% C I=1.23-1.60)$, progression-free survival (PFS) ( $H R=1.75$, 95\% CI=1.36-2.15), disease-specific survival (DSS) and cancer-specific survival (CSS) (HR=1.27, $95 \% \mathrm{CI}=1.19-1.35)$ in the bladder cancer patients. Additionally, an elevated pretreatment NLR suggested a worse OS rate in the $\mathrm{mUC}$ or UUC patients ( $\mathrm{HR}=1.63,95 \% \mathrm{CI}=1.34-1.91$ ). The pooled ORs and $95 \%$ CIs showed that a high pretreatment NLR could be a risk indicator for certain pathological features, such as lymphovascular invasion, a positive margin status and advanced tumor stage. Conclusions: our results showed that a high pretreatment NLR predicted poor prognosis in bladder cancer, mUC and UUC patients.
\end{abstract}

Ran Xu

KARGER
Department of Urology, The Second Xiangya Hospital Central South University, Hunan Province, (People's Republic of China) E-Mail xuran@csu.edu.cn 


\section{Introduction}

An estimated 79, 030 new bladder cancer cases will be diagnosed and an estimated 16, 870 cancer-related deaths will occur in the United States in 2017 [1]. Urothelial carcinoma is the most common type in bladder carcinomas, up to $95 \%$ of bladder malignancies are urothelial carcinomas, which are derived from the lining surface epithelium of urinary tract from the calyces to the distal ureter [2-4]. Nearly $75 \%$ of patients are diagnosed with nonmuscle-invasive bladder cancer (NMIBC), and the remaining patients have muscle-invasive bladder cancer (MIBC) or metastatic disease [5]. A transurethral resection of bladder tumor (TURBT) is the initial step for NMIBC patients, radical cystectomy (RC) is the gold standard therapy for MIBC patients, and cisplatin-based combination chemotherapy is the main treatment for metastatic urothelial carcinoma (mUC) or unresectable urothelial carcinoma (uUC) patients [5-7]. Despite developments in the management of bladder carcinomas, the outcomes have remained largely unchanged over the past three decades, and the decisionmaking process in the treatment of urothelial carcinoma is often challenging $[6,8]$. To maximize the positive effect for bladder cancer, mUC and uUC patients, it is imperative for us to focus on the identification of prognostic biomarkers for risk stratification and individual therapies.

Accumulating studies have reported that a systemic inflammation is associated with the development and progression of various tumors [9-11]. More recently, SIR-related biomarkers, such as C-reactive protein (CRP) [12], the lymphocyte-monocyte ratio (LMR) [13], and the platelet-lymphocyte ratio (PLR) [14], have been investigated as potential predictors in bladder cancer. The neutrophil-lymphocyte ratio (NLR) has also been investigated as an economic and readily available predictor for bladder tumors in a few studies [15-19].

Previous systematic reviews and meta-analyses have assessed the NLR as a prognostic indicator in upper urinary tract urothelial carcinoma (UUTUC) [20] or urothelial carcinomas [21]. Although UUTUC and urothelial carcinoma of the bladder share many features, they represent two different diseases with varying risk factors, biological behaviors and prognoses [4, 22]. Moreover, various tumor stages, surgical procedures and therapies can also influence the prognosis of bladder cancer, which may contribute to the heterogeneity among the studies included for pooled analysis. Therefore, our study was conducted to evaluate the prognostic role of the pretreatment NLR in bladder cancer patients treated with RC or TURBT and in mUC or uUC patients treated with systemic chemotherapy.

\section{Materials and Methods}

\section{Literature search}

We systematically searched Pubmed, Embase, and the Web of Science up to July 1st, 2017 for studies involving the pretreatment NLR along with bladder cancer and metastatic or unresectable urothelial carcinoma. The following search terms were used: (bladder cancer OR bladder carcinoma OR bladder tumor OR urothelial carcinoma of bladder OR transitional carcinoma of bladder OR metastatic urothelial carcinoma OR unresectable urothelial carcinoma) AND (NLR OR neutrophil to lymphocyte ratio OR neutrophillymphocyte ratio OR neutrophil lymphocyte ratio). References in the identified articles were searched for potential inclusion. Duplicate studies were removed using EndNote software version X7 (Thomas Reuters, New York City, NY, USA).

\section{Inclusion criteria}

We included studies if (1) the diagnosis of bladder cancer or metastatic urothelial carcinoma was confirmed by pathology, (2) the NLR was defined as the absolute neutrophil count divided by the absolute lymphocyte count and was derived from serum samples collected from patients before treatment, and (3) the studies investigated the prognostic role of the pretreatment NLR and provided sufficient information to extract HRs and 95\% CIs for the prognostic variables (OS, RFS, PFS, DSS/CSS). 


\section{Cellular Physiology Cell Physiol Biochem 2018;46:1352-1364 \begin{tabular}{l|l} 
and Biochemistry Published online: April 23, 2018 & $\begin{array}{l}\text { (c) } 2018 \text { The Author(s). Published by S. Karger AG, Basel } \\
\text { www.karger.com/cpb }\end{array}$ \\
\hline
\end{tabular}}

Wu et al.: NLR as a Predictor in BC, mUC or uUC

\section{Exclusion criteria}

Studies were excluded if they met the following criteria: (1) the published data were in reviews, letters, case reports, editorials, conference abstracts or animal studies; (2) the HRs and 95\% CIs were calculated using a continuous variable for the NLR; (3) there were duplicate or overlapping data; (4) the number of patients was <10; or (5) the studies were not relevant regarding the association between the pretreatment NLR and the prognostic variables (OS, RFS, PFS, DSS/CSS) in bladder cancer, mUC or uUC.

\section{Data synthesis and analysis}

The following data were extracted using a predefined form: first author's name (year of publication), study design, geographical area of patients, number of cases, diagnosis, cut-off value, prognostic variables and analysis methods (multivariate analysis or univariate analysis). The raw data reporting the association between the pretreatment NLR and pathological features in the included studies were also collected and used to calculate the ORs and related 95\% CIs with SPSS software, version 19 (International Business Machines Corporation, New York City, NY, USA). The pathological features included concomitant Cis (yes vs. no), lymphovascular invasion (yes vs. no), lymph node stage ( + vs. Nx, N0), lymph node involvement (yes vs. no), margin status (positive vs. negative), tumor stage ( $\geq \mathrm{T} 3 \mathrm{vs} .<\mathrm{T} 3$ ), tumor stage (T1 vs. Ta), multifocality (yes vs. no), tumor grade ( 3 vs. 1,2 ), tumor grade (high vs. low) and tumor size ( $\geq 3 \mathrm{~cm} \mathrm{vs.}<3 \mathrm{~cm}$ ).

The NOS system was adopted to evaluate the quality of the included studies [23]. The NOS system scores ranged from 0 to 9 and covered three general aspects: subject selection, comparability of groups, and clinical outcome. Both the data extraction and quality assessment of the included studies were reviewed by two reviewers independently, and any disagreement was addressed through discussion with a third reviewer.

\section{Statistical analysis}

The HRs and related 95\% CIs (high vs. low level of NLR) were extracted to assess the association between the pretreatment NLR and prognostic variables (OS, RFS, PFS and DSS/CSS). The ORs and related 95\% CIs were calculated to evaluate the correlation between the pretreatment NLR and the pathological characteristics of bladder cancer. The pooled HRs or ORs were analyzed using the Stata software program, version 12.0 (StatCorp, College Station, TX, USA). Heterogeneity among the included studies was assessed using the I-square $\left(\mathrm{I}^{2}\right)$ result and p-value. An $\mathrm{I}^{2}$ value $>50 \%$ or p-value $<0.05$ indicated the presence of significant heterogeneity. We preferred to calculate the pooled HRs or ORs using the random-effects model; otherwise, we chose the fixed-effects model. If a pooled HR was $>1$ and 1 was also not included in its $95 \%$ CI, this result suggested that a high pretreatment NLR significantly correlated with a worse outcome in bladder cancer, mUC or uUC patients.

To detect potential heterogeneity among the included studies, subgroup analyses and sensitivity analyses were conducted. Publication bias was assessed with Begg's funnel plot and Egger's linear regression $[24,25]$. If the result from a Begg's or Egger's test indicated the existence of significant publication bias, the trim and fill method was utilized to adjust the pooled HRs and related 95\% CIs [26, 27]. A difference with a p-value $<0.05$ was deemed statistically significant.

\section{Results}

\section{Literature search and study selection}

A total of 341 studies were initially identified using the search strategy detailed in the Materials and Methods section, and 212 studies remained after the removal of duplicates. After screening the titles and abstracts, 73 studies were excluded because the publications were one of the following: a review, letter, case report, editorial, or conference abstract. Therefore, 139 remaining studies were further evaluated for eligibility, and 118 studies were further excluded because they were animal studies, had no survival data with the pretreatment NLR, were without sufficient data to extract the HRs and 95\% confidence intervals (CIs), used a continuous variable as the cut-off value, or had overlapping patients or $<10$ patients. A total of 21 studies were finally included in this pooled analysis, of which 17 studies included 11, 262 bladder cancer patients treated with RC or TURBT [15-17, 2841] and 4 studies involved 862 mUC or uUC patients treated with systemic chemotherapy [42-45]. The flow diagram for the study selection process is depicted in Fig. 1. 


\section{Characteristics of the included studies}

The number of patients in the study populations ranged from 50 to 4198 . The bladder cancer patients underwent TURBT or RC, and systemic chemotherapy was chosen as the main therapy for the mUC or uUC patients. The NLR data were collected before surgery or chemotherapy in the included studies. The cut-off values for the NLR ranged from 2 to 5 , and the cut-off value was not specified in 3 studies [15, 35, 38]. In Mano's study, there were two cut-off values, one each to evaluate the prognostic role of the NLR in progression-free survival (PFS) and relapsefree survival (RFS) [31]. All the NLRs were derived from serum before treatment. Overall survival (OS) was investigated as the prognostic variable in 13 studies, of which 9 studies involved bladder cancer patients and 4 studies investigated mUC or uUC patients. The prognostic role of the pretreatment NLR regarding RFS, PFS and diseasespecific survival/cancer-specific survival (DSS/CSS) in bladder cancer patients was evaluated in 9, 7 and 9 studies, respectively. Of the 21 included studies, Hermanns's study reported

Fig. 1. Flow chart for the selection of included studies.

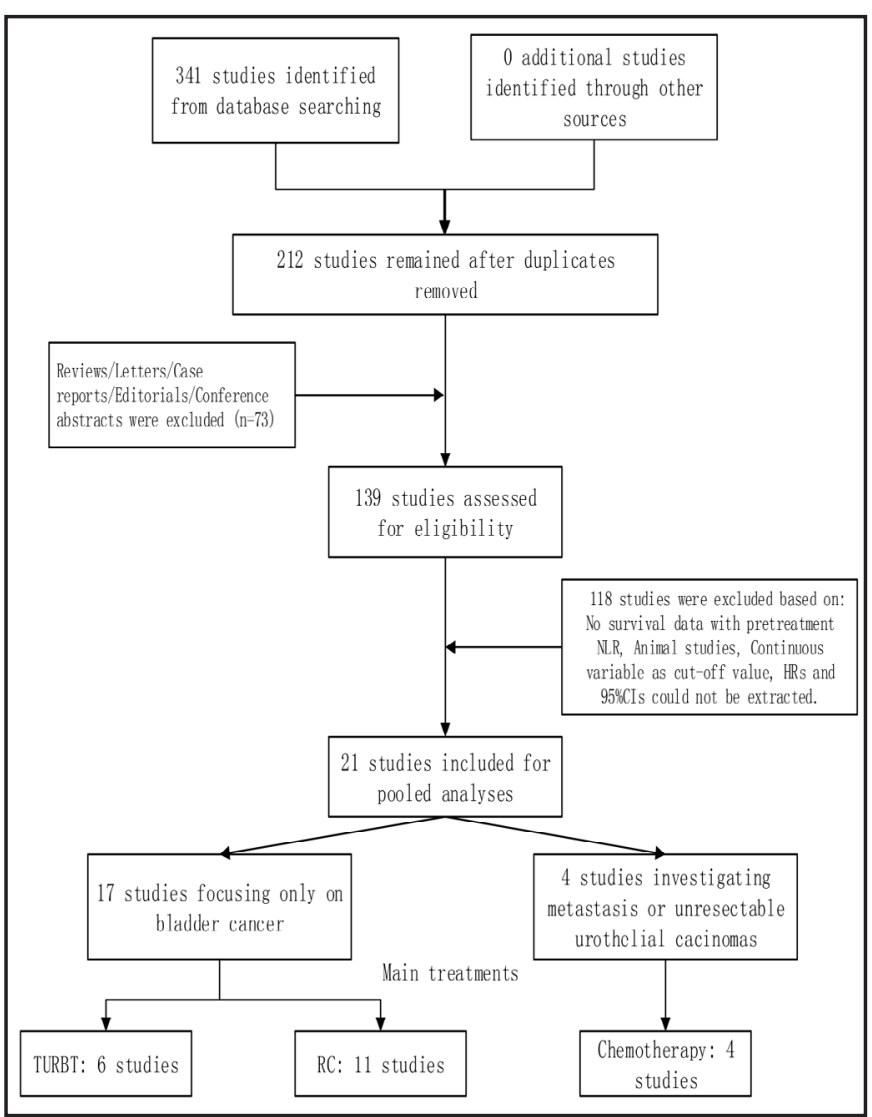
adjusted HRs and 95\% CIs [29],

Table 1. Characteristics of included studies. BC: bladder cancer; OS: overall survival; RFS: recurrence-free survival; PFS: progression-free survival; CSS: cancer-specific survival; DSS: disease-specific survival; RC: radical cystectomy; TURBT: transurethral resection of bladder tumor; M: multivariate analysis; U: univariate analysis; A: adjusted hazard ratio; mUC: metastatic urothelial carcinoma; uUC: unresectable urothelial carcinoma; NS: not specified

\begin{tabular}{|c|c|c|c|c|c|c|c|c|c|}
\hline $\begin{array}{c}\text { First author } \\
\text { (year) }\end{array}$ & Design & Area & $\begin{array}{c}\text { Sample } \\
\text { size }\end{array}$ & Diagnosis & Therapy & $\begin{array}{l}\text { Cut-off } \\
\text { value }\end{array}$ & Outcome & $\begin{array}{l}\text { Analysis } \\
\text { methods }\end{array}$ & $\begin{array}{c}\text { NOS } \\
\text { score }\end{array}$ \\
\hline Peng D et al [15] & Retrospective & China & 516 & $\mathrm{BC}$ & $\mathrm{RC}$ & NS & OS, PFS & M & 6 \\
\hline D'Andrea D et al. [16] & Retrospective & Austria & 4198 & $\mathrm{BC}$ & $\mathrm{RC}$ & 2.7 & RFS, CSS, OS & M & 8 \\
\hline Kang $M$ et al [17] & Retrospective & Korea & 385 & $\mathrm{BC}$ & $\mathrm{RC}$ & 2 & OS, CSS & M & 8 \\
\hline Gondo T et al. [28] & Retrospective & Japan & 189 & $\mathrm{BC}$ & $\mathrm{RC}$ & 2.5 & DSS & $\mathrm{M}$ & 8 \\
\hline Hermanns T et al. [29] & Retrospective & Canada & 424 & $\mathrm{BC}$ & $\mathrm{RC}$ & 3 & RFS, CSS, OS & A & 8 \\
\hline Ozcan C et al [30] & Retrospective & Turkey & 286 & $\mathrm{BC}$ & RC & 2.5 & DSS & M & 8 \\
\hline Mano R et al. [31] & Retrospective & Israel & 107 & $\mathrm{BC}$ & TURBT & $2.41 ; 2.43$ & PFS, RFS & $\mathrm{M}$ & 7 \\
\hline Morizawa Y et al. [32] & Retrospective & Japan & 110 & $\mathrm{BC}$ & $\mathrm{RC}$ & 2.6 & RFS, CSS, OS & M & 7 \\
\hline Favilla $\mathrm{V}$ et al [33] & Prospective & Italy & 178 & $\mathrm{BC}$ & TURBT & 3 & RFS, PFS & M & 8 \\
\hline Mbeutcha A et al. [34] & Retrospective & Austria & 1117 & $\mathrm{BC}$ & TURBT & 2.5 & RFS, PFS & M & 8 \\
\hline Bhindi B et al. [35] & Retrospective & Canada & 418 & $\mathrm{BC}$ & $\mathrm{RC}$ & NS & RFS, CSS, OS & M & 7 \\
\hline Kawahara T et al. [36] & Retrospective & Japan & 74 & $\mathrm{BC}$ & $\mathrm{RC}$ & 2.38 & os & M & 7 \\
\hline Buisan O et al. [37] & Retrospective & Spain & 50 & $\mathrm{BC}$ & $\mathrm{RC}$ & 5 & PFS, CSS, OS & M & 7 \\
\hline Hirasawa Y et al [38] & Retrospective & Japan & 136 & $\mathrm{BC}$ & $\mathrm{RC}$ & NS & CSS & M & 6 \\
\hline Ogihara K et al [39] & Retrospective & Japan & 605 & $\mathrm{BC}$ & TURBT & 2.2 & RFS, PFS & M & 8 \\
\hline Kang $M$ et al [40] & Retrospective & Korea & 1551 & $\mathrm{BC}$ & TURBT & 2 & OS & M & 8 \\
\hline D'Andrea D et al. [41] & Retrospective & Austria & 918 & $\mathrm{BC}$ & TURBT & 3 & RFS, PFS & M & 8 \\
\hline Bambury RM et al. [42] & Retrospective & USA & 129 & $\mathrm{mUC}$ & Chemotherapy* & $2.5 ; 3$ & OS & $\mathrm{U}$ & 7 \\
\hline Taguchi S et al. [43] & Retrospective & Japan & 185 & $\mathrm{mUC}$ & Chemotherapy & 3 & CSS, os & M & 6 \\
\hline Rossi L et al. [44] & Retrospective & UK & 292 & mUC, uUC & Chemotherapy & 3 & PFS, OS & $\mathrm{U}$ & 7 \\
\hline Su Y et al. [45] & Retrospective & Taiwan & 256 & mUC, uUC & Chemotherapy & 3 & OS & $\mathrm{U}$ & 7 \\
\hline
\end{tabular}




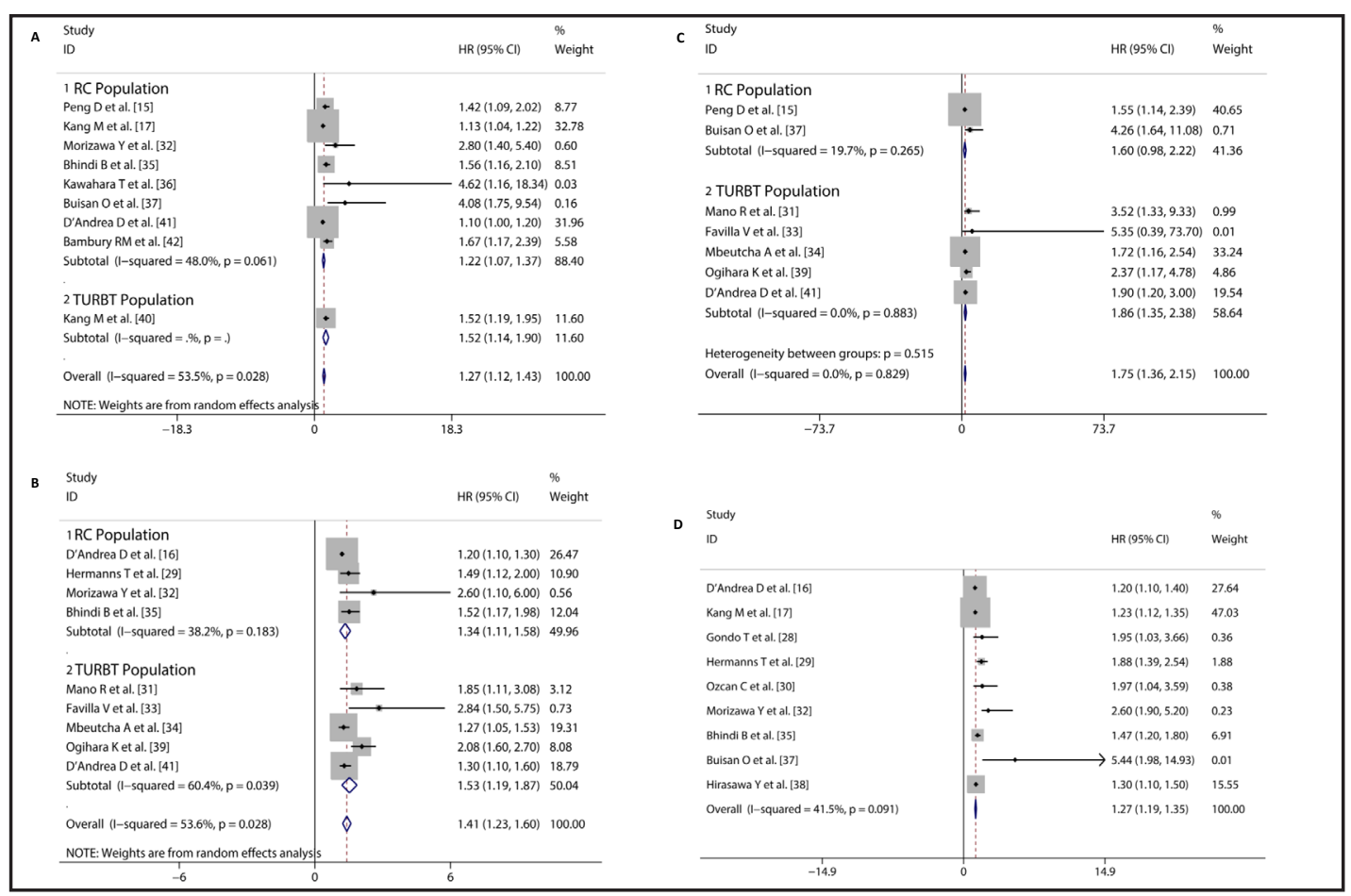

Fig. 2. Forest plot evaluating the prognostic role of pretreatment NLR with OS (A), RFS (B), PFS (C) and DSS/CSS (D) in bladder cancer patients. RC: radical cystectomy; TURBT: transurethral resection of bladder tumor.

while 3 studies reported HRs and 95\% CIs derived only from a univariate analysis [42, 44, 45]; the remaining studies presented HRs and 95\% CIs obtained by a multivariate analysis. With regard to the quality assessment of the included studies, the Newcastle-Ottawa Scale (NOS) scores ranged from 6 to 8 . The detailed characteristics are shown in Table 1.

\section{Prognostic significance of NLR for OS in bladder cancer patients}

Nine studies comprising 7726 patients presented the survival data regarding the pretreatment NLR and OS in bladder cancer patients. Overall, the pooled result derived from the random-effects model indicated that an elevated pretreatment NLR was significantly correlated with reduced OS (HR=1.27, 95\% CI=1.12-1.43) (Fig. 2A). Next, we performed a subgroup analysis according to the surgical procedures with patients divided into RC or TURBT populations, and the pooled results also suggested that the pretreatment NLR was associated with a worse OS rate regardless of the different surgical procedures. To identify potential heterogeneity, we further conducted subgroup analyses according to the sample size ( $>400$ and $<400)$, stratified cut-off values $(\geq 3,<3$ and not specified), and geographical area of the patients (Asian and non-Asian). A significant association between a high pretreatment NLR and reduced OS was observed in all the subgroup analyses, which is consistent with the overall pooled result. The detailed results from the subgroup analyses of the pretreatment NLR and OS are summarized (for all online suppl. material, see www. karger.com/doi/ 10.1159/000489152) in Suppl. Table S1.

\section{Pretreatment NLR and OS in mUC or UUC patients}

Four studies reported the prognostic data for the NLR and OS in 862 mUC or uUC patients mainly treated with systemic chemotherapy. The pooled HRs and 95\% CIs indicated that a high pretreatment NLR was correlated with a worse OS rate in mUC or uUC patients ( $\mathrm{HR}=1.63,95 \% \mathrm{CI}=1.34-1.91$ ) (Fig. 3). No significant heterogeneity was found among the included studies $\left(\mathrm{I}^{2}=0.0 \%, \mathrm{p}=0.924\right)$ (Fig. 3 ).

\section{KARGER}




\section{Cellular Physiology Cell Physiol Biochem 2018;46:1352-1364

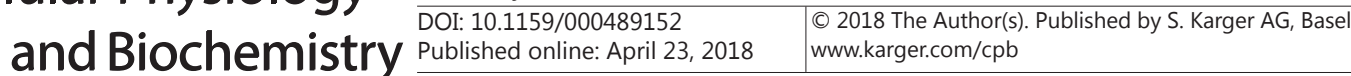 Wu et al.: NLR as a Predictor in BC, mUC or uUC}

Prognostic value of NLR for RFS in bladder cancer patients

of the included studies, 9 studies comprising 8075 patients evaluated the association between an elevated pretreatment NLR and RFS in bladder cancer patients. According to the overall pooled result, an elevated pretreatment NLR was associated with a shorter RFS time in bladder cancer patients $(\mathrm{HR}=1.41$, 95\% CI=1.23-1.60) (Fig. 2B). Furthermore, we conducted subgroup analyses to explore potential heterogeneity, and the results from the subgroup analyses indicated a significant correlation between a high pretreatment NLR and reduced RFS in bladder cancer patients (see online suppl. material, Suppl. Table S2). Regarding the two main surgical procedures for bladder cancer patients, both of the pooled HRs suggested that high pretreatment NLRs were correlated with worse RFS rates in both the TURBT population (HR $=1.53,95 \%$ $\mathrm{CI}=1.19-1.87)$ and the $\mathrm{RC}$ population $(\mathrm{HR}=1.23,95 \% \mathrm{CI}=1.14-1.33)$ (see online suppl. material, Suppl. Table S2).

\section{Effect of NLR on PFS in bladder cancer patients}

The correlation between the pretreatment NLR and PFS was reported in 7 studies involving 3491 bladder cancer patients. The combined analysis showed that a high pretreatment NLR was significantly associated with a poorer PFS in bladder cancer patients ( $\mathrm{HR}=1.75,95 \% \mathrm{CI}=1.36-2.15$ ) (Fig. $2 \mathrm{C}$ ), without significant heterogeneity $\left(\mathrm{I}^{2}=0 \%\right.$, $\left.\mathrm{p}=0.829\right)$. When we divided the included patients into TURBT (2925 patients) and RC (566 patients) populations, the result was similar in the TURBT population $(\mathrm{HR}=1.86,95 \% \mathrm{CI}=1.35-2.38)$, while the pooled result from the RC population indicated no significant association between the NLR and RFS (HR=1.60, 95\% CI=0.98-2.22). Regarding the subgroup analyses of the sample size ( $>400$ and $<400$ ), geographical area of the patients (Asian and non-Asian) and different cut-off values ( $\geq 3,<3$ and not specified), all the results demonstrated that a high pretreatment NLR predicted poorer PFS in bladder cancer patients. The details are shown (see online suppl. material) in Suppl. Table S3.

\section{Prognostic role of NLR regarding DSS/CSS in bladder cancer patients}

Nine studies comprising 6196 patients provided the survival data to assess the pretreatment NLR in relation to DSS or CSS in bladder cancer patients. All the included patients were treated with RC. The overall result suggested that a high pretreatment NLR was significantly associated with worse DSS/CSS in bladder cancer patients (HR=1.27, 95\% $\mathrm{CI}=1.19-1.35$ ) (Fig. 2D). A significant correlation was also found in the subgroup analyses detailed (see online suppl. material) in Suppl. Table S4.

\section{$N L R$ in the risk assessment of pathological characteristics in bladder cancer patients}

Ten included studies reported the relationship between the pretreatment NLR and the pathological features in bladder cancer patients. The pooled odds ratios (ORs) and $95 \%$ CIs suggested that a high pretreatment NLR was a potential predictor of the risk of lymphovascular invasion (OR=1.38, 95\% $\mathrm{CI}=1.21-1.55)$, positive margin status $(\mathrm{OR}=1.13$, 95\% CI=1.02-1.24), advanced tumor stage ( $\geq \mathrm{T} 3$ vs. $<\mathrm{T} 3)(\mathrm{OR}=1.54,95 \% \mathrm{CI}=1.36-1.71)$, tumor stage (T1 vs. Ta) $(\mathrm{OR}=1.45,95 \% \mathrm{CI}=1.19-1.71)$ and tumor grade $(3$ vs. 1,2$)(\mathrm{OR}=1.78$, 
Wu et al.: NLR as a Predictor in BC, mUC or uUC

Table 2. The association between NLR and pathological characteristics (NLR: high vs. low)

\begin{tabular}{|c|c|c|c|c|c|c|c|}
\hline \multirow{2}{*}{ Patient characteristics } & \multirow{2}{*}{$\begin{array}{l}\text { Number of } \\
\text { studies }\end{array}$} & \multirow{2}{*}{$\begin{array}{l}\text { Number of } \\
\text { studies }\end{array}$} & \multirow{2}{*}{$\begin{array}{l}\text { Effects } \\
\text { model }\end{array}$} & \multirow{2}{*}{ OR $(95 \% \mathrm{CI})$} & \multicolumn{3}{|c|}{ Heterogeneity } \\
\hline & & & & & $\mathrm{Chi}^{2}$ & $\mathrm{I}^{2}$ & $\mathrm{p}$ \\
\hline Concomitant Cis (yes vs. no) & 7 & 7547 & Fixed & $0.92(0.82-1.02)$ & 11.54 & 48 & 0.073 \\
\hline Lymphovascular invasion (yes vs. no) & 4 & 4782 & Fixed & $1.38(1.21-1.55)$ & 1.1 & 0 & 0.776 \\
\hline Lymph node stage (N+vs. Nx, N0) & 2 & 474 & Fixed & $0.84(0.48-1.21)$ & 0.39 & 0 & 0.535 \\
\hline Lymph node involvement (yes vs. no) & 3 & 4382 & Random & $1.47(0.57-2.36)$ & 4.47 & 55.2 & 0.107 \\
\hline Margin status (positive vs. negative) & 4 & 658 & Fixed & $1.13(1.02-1.24)$ & 1.57 & 0 & 0.667 \\
\hline Tumor stage $(\geq \mathrm{T} 3$ vs. $<\mathrm{T} 3)$ & 5 & 4856 & Fixed & $1.54(1.36-1.71)$ & 3.48 & 0 & 0.481 \\
\hline Tumor stage (T1 vs. Ta) & 5 & 2523 & Fixed & $1.45(1.19-1.71)$ & 1.71 & 0 & 0.789 \\
\hline Tumor multifocality (yes vs. no) & 5 & 2945 & Fixed & $0.92(0.76-1.09)$ & 6.28 & 36.3 & 0.179 \\
\hline Tumor grade ( 3 vs. 1,2 ) & 4 & 6340 & Random & $1.78(1.12-2.44)$ & 15.19 & 80.2 & 0.002 \\
\hline Tumor grade (high vs. low) & 2 & 228 & random & $1.23(-0.10-2.55)$ & 3.11 & 67.9 & 0.078 \\
\hline Tumor size $(\geq 3 \mathrm{~cm}$ vs. $<3 \mathrm{~cm})$ & 5 & 2925 & fixed & $1.11(0.90-1.32)$ & 1.85 & 0 & 0.762 \\
\hline
\end{tabular}
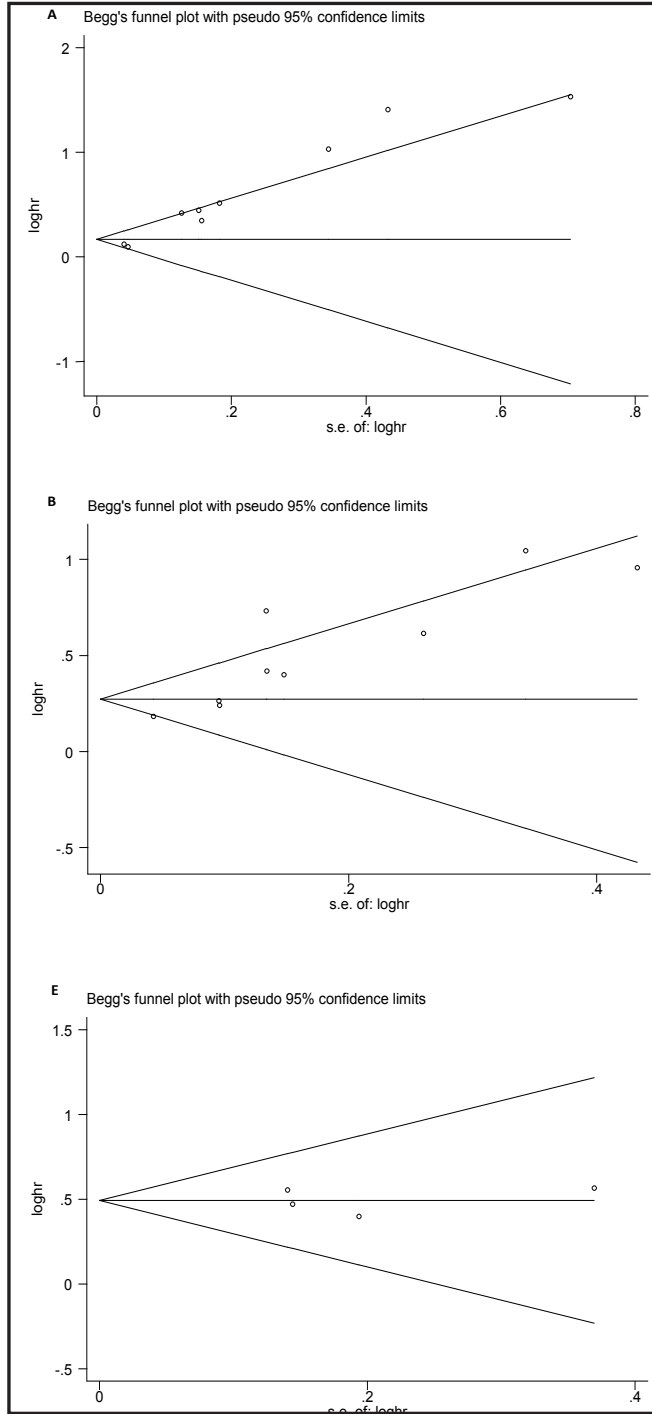

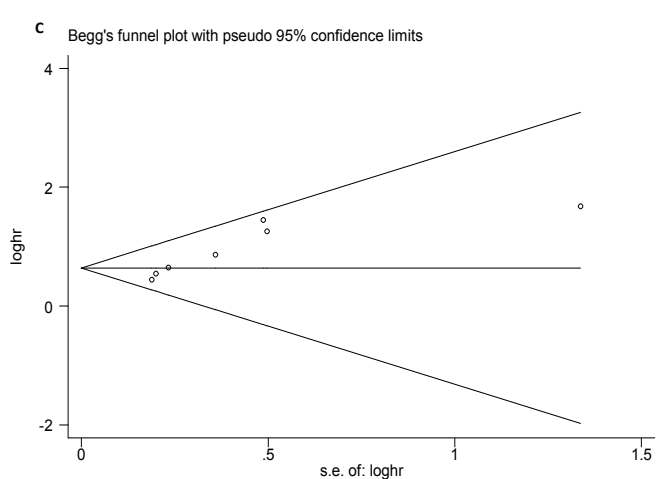

D Begg's funnel plot with pseudo $95 \%$ confidence limits

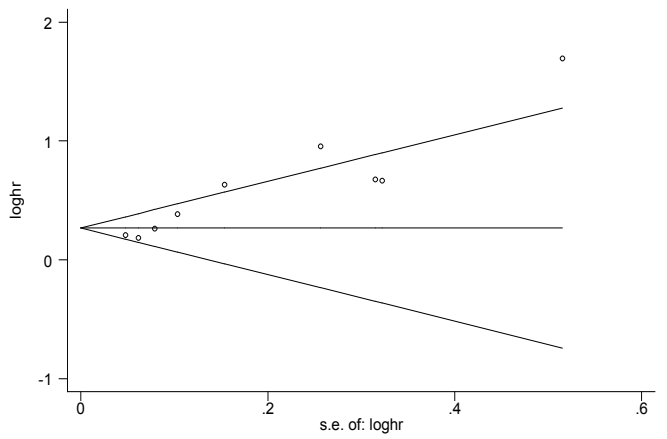

Fig. 4. Begg's funnel plots assessing the potential publication bias among the included studies: pretreatment NLR with OS (A) ( $p=0.048)$, RFS (B) ( $p=0.048)$, PFS (C) ( $=0.035)$, and DSS/CSS (D) $(p=0.009)$ in bladder cancer patients; pretreatment NLR with OS in metastatic or unresectable urothelial carcinoma patients (E) $(\mathrm{p}=0.734)$.

95\% CI=1.12-2.44), but there was no significant correlation with concomitant carcinoma in situ (Cis), lymph node involvement, lymph node stage, tumor multifocality or tumor size. The results are detailed in Table 2. 


\section{Cellular Physiology Cell Physiol Biochem 2018;46:1352-1364 \begin{tabular}{l|l} 
DOI: 10.1159/000489152 & $\begin{array}{l}\text { O 2018 The Author(s). Published by S. Karger AG, Basel } \\
\text { www.karger.com/cpb }\end{array}$
\end{tabular} \\ Wu et al.: NLR as a Predictor in BC, mUC or uUC}

\section{Sensitivity analysis}

We performed leave-one-out sensitivity analyses by removing each study in turn to evaluate the impact of any single study on the pooled results. No significant alteration was observed for the HRs and related 95\% CIs concerning OS, PFS or DSS/CSS. However, regarding RFS in bladder cancer patients, the sensitivity analysis indicated that D'Andrea's study with an RC population was the main source of heterogeneity (see online suppl. material, Suppl. Fig. S1B). After removing the abovementioned study, the heterogeneity was obviously decreased. However, the corresponding pooled HR and 95\% CI still suggested that a high preoperative NLR was associated with reduced RFS (HR=1.41, 95\% CI=1.27-1.55) (see online suppl. material, Suppl. Fig. S2). Collectively, the results from the sensitivity analyses indicated that our findings were relatively stable.

\section{Evaluation of publication bias}

Begg's funnel plots indicated significant asymmetry concerning the NLR with OS ( $p=0.048$, Fig. 4A), NLR with RFS ( $\mathrm{p}=0.048$, Fig. 4B), NLR with PFS ( $\mathrm{p}=0.035$, Fig. 4C) and NLR with DSS/CSS ( $p=0.009$, Fig. 4D) in bladder cancer patients, but no substantial symmetry was found for the NLR with OS in the mUC or uUC patients ( $p=0.734$, Fig. 4D). Moreover, the results from Egger's test were similar to those from Begg's test (see online suppl. material, data shown in Suppl. Fig. S3). For the analyses with significant publication bias, we further performed "trim and fill" analyses. The "trim and fill" results suggested that there were 5, 5, 3 and 4 unpublished studies assessing the pretreatment NLR with OS, RFS, PFS and DSS/CSS in bladder cancer patients, respectively (see online suppl. material, Suppl. Fig. S4A-D). Meanwhile, the "trim and fill" result also indicated there was no unpublished studies assessing the pretreatment NLR with OS in the mUC or uUC patients (see online suppl. material, Suppl. Fig. S4E). However, the results from the adjusted models using the "trim and fill" method were consistent with our primary pooled results; the details are shown (see online suppl. material) in Suppl. Table S5.

\section{Discussion}

Chronic inflammation is involved in the development and progression of invasive and metastatic bladder cancer [46]. Immune cells play multifaceted roles in the tumor microenvironment via dynamic and extensive communication with cancer cells [47]. Neutrophils can contribute to chronic inflammation and promote tumor angiogenesis and growth by secreting specific molecules $[46,48]$. A deficiency in or disruption of lymphocytes is involved in the SIR and promotion of malignancies [13,47]. The NLR, calculated as the absolute neutrophil count divided by the absolute lymphocyte count, has often been investigated as a potential indicator in various tumors [49-51]. Bladder cancer is still a challenging issue, especially the muscle-invasive and metastatic diseases, and efforts to explore and validate prognostic biomarkers for use in the personalized treatment of bladder cancer, mUC or uUC carcinoma patients are urgently required $[5,6,52]$.

In this pooled analysis, 17 studies were included to evaluate the prognostic role of the pretreatment NLR with the following prognostic variables: OS, RFS, PFS, and DSS/CSS in bladder cancer patients. Our primary results indicated that an elevated pretreatment NLR significantly correlated with reduced OS, RFS, PFS, and DSS/CSS in bladder cancer patients. Regarding the surgical procedures, the included patients were divided into a TURBT population and an RC population, and the pooled results suggested that a high pretreatment NLR predicted reduced OS, RFS and DSS/CSS in bladder cancer patients regardless of treatment with TURBT or RC. A high pretreatment NLR predicted a shorter PFS time in the TURBT population, while no significant association was found in the RC population; however, this result should be cautiously interpreted due to the limited studies and patients in the RC population. To further explore potential heterogeneity among the included studies, subgroup analyses were conducted, and a high pretreatment NLR was also indicated as a potential prognostic biomarker for bladder cancer patients, regardless of the cut-off values 
or geographical area of the patients.

Sometimes, therapeutic strategies may be difficult to determine due to the insufficient information available before treatment. To improve the management of bladder cancer, it is urgent that new pretreatment risk stratification models for multimodal treatment strategies are developed in bladder cancer patients [28]. Therefore, we assessed the association between the pretreatment NLR and pathological characteristics, and our pooled results demonstrated that the pretreatment NLR could be a significant indicator for lymphovascular invasion (yes vs. no), margin status (positive vs. negative), tumor stage ( $\geq \mathrm{T} 3 \mathrm{vs}$. $<\mathrm{T} 3$ or T1 vs. Ta) and tumor grade (3 vs. 1, 2). However, no significant association could be found between the pretreatment NLR and concomitant Cis, lymph node involvement, lymph node stage, tumor multifocality or tumor size, which were similar to the pooled results in urothelial carcinoma but differed to some extent [21].

Urothelial carcinoma is the most common type of bladder cancer [3]. Conducting surgery is the first choice for organ-confined bladder malignancies. Cisplatin-based systemic chemotherapy has been recommended as standard care for mUC or uUC patients, and novel agents alone or in combination are in development for patients with cisplatin-resistant mUC or uUC $[42,53,54]$. However, mUC or uUC is still an intractable disease with a poor prognosis. Emerging studies have evaluated the prognostic value of the NLR in mUC or uUC patients, and there have been conflicting results [42-45]. In this context, we performed our study to evaluate the prognostic role of the pretreatment NLR in MUC or uUC patients treated with systemic chemotherapy, and the pooled result indicated that an elevated pretreatment NLR predicted worse OS in mUC or uUC patients with no significant heterogeneity among the included studies, which suggests that the pretreatment NLR is a prognostic indicator for mUC or uUC patients.

To avoid heterogeneity among the studies in this pooled analysis, we only included those studies with stratified or unspecified cut-off values for the NLR. Some studies that used a continuous variable for the NLR also reported a correlation between a high pretreatment NLR and a poor prognosis in bladder cancer, mUC or uUC patients [42, 55]. Moreover, derived-NLR (dNLR), calculated as the absolute neutrophil count/(white blood cell count - neutrophil count), has also been investigated as a prognostic indicator in bladder cancer patients [56]. However, additional large-scale studies are still required to verify the prognostic role of NLR-derived biomarkers and to determine the optimal cut-off values for the NLR or dNLR.

More recently, Tang et al. has published a meta-analysis about the clinical use of NLR in bladder cancer patients, and their results also indicated high NLR was associated with poor prognosis in bladder cancer patients [57]. However, there are still many different points in our manuscript, compared to Tang et al's study. Firstly, we included the eligible studies up to July, 2017, there are more included studies for pooled analysis in our manuscript. Second, in order to make the included studies more comparable, we just focused on the NLR derived from "serum before treatment". Secondly, in addition to evaluate the role of NLR with primary prognostic outcomes (OS, RFS, CSS/DSS, PFS), We also have conducted comprehensive assessment of the association between pretreatment NLR and the pathological characteristics in bladder cancer patients. Moreover, our pooled analysis was conducted to focus on the prognostic role of the pretreatment NLR in bladder cancer, mUC or uUC patients. Subgroup analyses according to the different treatments that patients received were also performed to validate our results. Last but not the least, comparing to Tang et al's study, we have used more detailed and in-depth methods, such as sensitivity analysis, "trim and fill" analysis to explore the potential heterogeneity and validate our conclusions. However, certain limitations to our study still existed. First, most of the included studies were designed as a retrospective studies, which could contribute to potential heterogeneity in patient selection. Second, substantial heterogeneity was found among the included studies regarding analyses of the NLR and OS, RFS and DSS/CSS. Therefore, we performed subgroup analyses and sensitivity analyses to explore this heterogeneity, and the results confirmed our primary findings. Third, significant publication bias could be identified among the studies of OS, RFS, PFS 


\section{Cellular Physiology Cell Physiol Biochem 2018;46:1352-1364 and Biochemistry Published online: April 23, $2018 \quad \begin{aligned} & \text { DOI 1018 2018 The Author(s). Published by S. Karger AG, Basel } \\ & \text { www.karger.com/cpb }\end{aligned}$ \\ Wu et al.: NLR as a Predictor in BC, mUC or uUC}

and DSS/CSS in bladder cancer patients according to Begg's and Egger's tests. Most of the included studies reported that an elevated pre-treatment NLR significantly correlated with a poor prognosis in bladder cancer patients, and some studies with neutral results were not included due to insufficient data $[58,59]$, which may contribute to the publication bias. Trim and fill analyses were performed to estimate the missing publications, and the filled analyses validated our results in both fixed- and random-effects models. Additionally, nearly all the included studies reported multivariate HRs and 95\% CIs. However, three studies involving mUC or uUC patients reported only univariate HRs and 95\% CIs [42, 44, 45], which may have overestimated the prognostic role of the pretreatment NLR.

\section{Conclusion}

Our results demonstrated that the pretreatment NLR is a prognostic indicator for bladder cancer, mUC and uUC patients. An elevated pretreatment NLR was associated with reduced OS, RFS and DSS/CSS in bladder cancer patients regardless of whether they were treated with TURBT or RC. A high pretreatment NLR predicted a shorter PFS time in the TURBT population, while no significant association was found in the RC population. For mUC or uUC patients treated with systemic chemotherapy, a high pretreatment NLR indicated a worse OS rate. Additionally, the pretreatment NLR could be a risk predictor for pathological features. Collectively, the pretreatment NLR is an economic and practical biomarker and has potential value for clinical decision-making and personalized therapy for bladder cancer, mUC and uUC patients. Well-designed prospective studies are still necessary to validate our findings in the future.

\section{Abbreviations}

NLR (neutrophil-lymphocyte ratio); dNLR (derived neutrophil-lymphocyte ratio); SIR (systemic inflammatory response); BC (bladder cancer); mUC (metastatic urothelial carcinoma); uUC (unresectable urothelial carcinoma); Cis (carcinoma in situ); RC (radical cystectomy); TURBT (transurethral resection of bladder tumor); OS (overall survival); RFS (relapse-free survival); PFS (progression-free survival); CSS (cancer-specific survival); DSS (disease-specific survival); NOS (Newcastle-Ottawa Scale); HR (hazard ratio); OR (odds ratio); 95\% CI (95\% confidence interval)

\section{Acknowledgements}

Shuiqing Wu is sponsored by China Scholarship Council (\#201606370183) as a visiting researcher at the University of Edinburgh. The funder (China Scholarship Council) had no role in the study design, data collection and analysis, decision to publish, or preparation of the manuscript.

This study was supported by the grant from Science and Technology Agency of Hunan Province (no. 2016JJ3178) and Finance Department of Hunan Province (2016-129). The funders had no role in the study design, data collection and analysis, decision to publish, or preparation of the manuscript.

\section{Disclosure Statement}

The authors declare that they have no conflicts of interests. 


\section{Cellular Physiology Cell Physiol Biochem 2018;46:1352-1364 and Biochemistry Published online: April 23, $2018 \quad \begin{aligned} & \text { DOI: 10.1159/000489152 } 2018 \text { The Author(s). Published by S. Karger AG, Basel } \\ & \text { www.karger.com/cpb }\end{aligned}$}

Wu et al.: NLR as a Predictor in BC, mUC or uUC

\section{References}

1 Siegel RL, Miller KD, Jemal A. Cancer statistics, 2017 CA Cancer J Clin 2017;67:7-30.

2 Wu S, Chen L, Wan Q, Zhang L, Zhao X, Tang X. Inflammatory myofibroblastic tumor of the urinary bladder in a patient with the left renal cell carcinoma: A case report. Exp Ther Med 2014;7:1010-1012.

-3 Reuter VE, Grossman HB, Blute ML, Soloway MS, Dinney CPN, Liou LS, Jones JS. The pathology of bladder cancer. Urology 2006;67:11-18.

4 Miyazaki J, Nishiyama H. Epidemiology of urothelial carcinoma. Int J Urol 2017;24:1-5.

5 Kamat AM, Hahn NM, Efstathiou JA, Lerner SP, Malmström PU, Choi W, Guo CC, Lotan Y, Kassouf W. Bladder cancer. Lancet 2016;6736:1-15.

-6 Lobo N, Mount C, Omar K, Nair R, Thurairaja R, Khan MS. Landmarks in the treatment of muscle-invasive bladder cancer. Nat Rev Urol 2017;14:565-574.

7 Abufaraj M, Gust K, Moschini M, Foerster B, Soria F, Mathieu R, Shariat SF. Management of muscle invasive, locally advanced and metastatic urothelial carcinoma of the bladder: a literature review with emphasis on the role of surgery. Transl Androl Urol 2016;5:735-744.

8 Stein JP, Skinner DG. Radical cystectomy for invasive bladder cancer: Long-term results of a standard procedure. World J Urol 2006;24:296-304.

-9 Gakis G, Todenhöfer T, Stenzl A. The prognostic value of hematological and systemic inflammatory disorders in invasive bladder cancer. Curr Opin Urol 2011;21:428-433.

10 Schepisi G, Santoni M, Massari F, Gurioli G, Salvi S, Conteduca V, Montironi R, De Giorgi U. Urothelial Cancer: Inflammatory Mediators and Implications for Immunotherapy. BioDrugs 2016;30:263-273.

-11 Wu S, Xu R, Wan Q Zhu X, Zhang L, Jiang H, Zhao X. Assessment of the potential diagnostic role of anaplastic lymphoma kinase for inflammatory myofibroblastic tumours: A meta-analysis. PLoS One 2015;10:1-12.

$>12$ Zhou L, Cai X, Liu Q, Jian Z, Li H, Wang K. Prognostic Role of C-Reactive Protein In Urological Cancers : A Meta-Analysis. Sci Rep 2015;5:12733.

$>13$ Song W, Tian C, Wang K, Zhang R, Zou S. The pretreatment lymphocyte to monocyte ratio predicts clinical outcome for patients with hepatocellular carcinoma: A meta-analysis. Sci Rep 2017;7:46601.

14 Wu S, Wan Q Xu R, Zhu X, He H ZX. Systematic review and meta-analysis of the prognostic value of preoperative platelet-to-lymphocyte ratio in patients with urothelial carcinoma. Oncotarget 2017;8:91694-91702.

15 Peng D, Gong Y-Q, Hao H, He Z-S, Li X-S, Zhang C-J, Zhou L-Q. Preoperative Prognostic Nutritional Index is a Significant Predictor of Survival with Bladder Cancer after Radical Cystectomy: a retrospective study. BMC Cancer 2017;17:391.

16 D’Andrea D, Moschini M, Gust KM, Abufaraj M, Özsoy M, Mathieu R, Soria F, Briganti A, Rouprêt M, Karakiewicz PI, Shariat SF. Lymphocyte-to-monocyte ratio and neutrophil-to-lymphocyte ratio as biomarkers for predicting lymph node metastasis and survival in patients treated with radical cystectomy. J Surg Oncol 2017;115:455-461.

17 Kang M, Jeong CW, Kwak C, Kim HH, Ku JH. The Prognostic Significance of the Early Postoperative Neutrophil-to-Lymphocyte Ratio in Patients with Urothelial Carcinoma of the Bladder Undergoing Radical Cystectomy. Ann Surg Oncol 2016;23:335-342.

18 Zheng J, Cai J, Li H, Zeng K, He L, Fu H, Zhang J, Chen L, Yao J, Zhang Y, Yang Y. Neutrophil to Lymphocyte Ratio and Platelet to Lymphocyte Ratio as Prognostic Predictors for Hepatocellular Carcinoma Patients with Various Treatments: a Meta-Analysis and Systematic Review. Cell Physiol Biochem 2017;44:967-981.

19 Huang Q, Zhou L, Zeng W, Ma Q, Wang W, Zhong M, Yu Y. Prognostic Significance of Neutrophil-toLymphocyte Ratio in Ovarian Cancer: A Systematic Review and Meta-Analysis of Observational Studies. Cell Physiol Biochem 2017;41:2411-2418.

20 Marchioni M, Cindolo L, Autorino R, Primiceri G, Arcaniolo D, De Sio M, Schips L. High Neutrophil-tolymphocyte Ratio as Prognostic Factor in Patients Affected by Upper Tract Urothelial Cancer: A Systematic Review and Meta-analysis. Clin Genitourin Cancer 2017;15:343-349.e1.

21 Li X, Ma X, Tang L, Wang B, Chen L, Zhang F. Prognostic value of neutrophil-to-lymphocyte ratio in urothelial carcinoma of the upper urinary tract and bladder : a systematic review and meta-analysis . Oncotarget 2016;8: 62681-62692.

22 Green DA, Rink M, Xylinas E, Matin SF, Stenzl A, Roupret M, Karakiewicz PI, Scherr DS, Shariat SF. Urothelial carcinoma of the bladder and the upper tract: Disparate twins. J Urol 2013;189:1214-1221. 


\section{Cellular Physiology Cell Physiol Biochem 2018;46:1352-1364

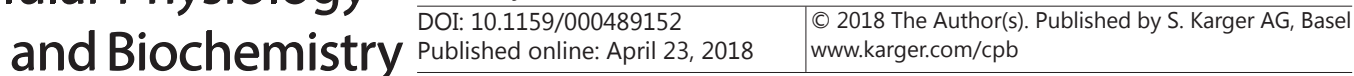

Wu et al.: NLR as a Predictor in BC, mUC or uUC

23 Wells G, Shea B, O'Connell D, Peterson J, Welch V, Losos M,Tugwell P. Newcastle -Ottawa Quality Assessment Scale Case Control Studies. Ottawa Hospital Research Institute 2013; http://www.ohri.ca/programs/ clinical_epidemiology/oxford.asp.

24 Begg CB, Mazumdar M. Operating Characteristics of a Rank Correlation Test for Publication Bias. Biometrics 1994;50:1088-101.

25 Egger M, Davey Smith G, Schneider M, Minder C. Bias in meta-analysis detected by a simple, graphical. Bmj 1997;315:629-634.

-26 Duval S, Tweedie R. Trim and Fill: A Simple Funnel-Plot-Based Method of Testing and Adjusting for Publication Bias in Meta-Analysis. Biometrics 2000;56:455-463.

-27 Zhou D, Gong H, Tan C, Luo J. Prognostic significance of anti-diabetic pancreatic cancer : A meta-analysis. Oncotarget 2017;8:62349-62357.

28 Gondo T, Nakashima J, Ohno Y, Choichiro O, Horiguchi Y, Namiki K, Yoshioka K, Ohori M, Hatano T, Tachibana M. Prognostic value of neutrophil-to-lymphocyte ratio and establishment of novel preoperative risk stratification model in bladder cancer patients treated with radical cystectomy. Urology 2012;79:1085-1091.

29 Hermanns T, Bhindi B, Wei Y, Yu J, Noon AP, Richard PO, Bhatt JR, Almatar A, Jewett MAS, Fleshner NE, Zlotta AR, Templeton AJ, Kulkarni GS. Pre-treatment neutrophil-to-lymphocyte ratio as predictor of adverse outcomes in patients undergoing radical cystectomy for urothelial carcinoma of the bladder. Br J Cancer 2014;111:444-451.

-30 Ozcan C, Telli O, Ozturk E, Suer E, Gokce MI, Gulpinar O, Oztuna D, Baltaci S, Gogus C. original The prognostic significance of preoperative leukocytosis and neutrophil-to-lymphocyte ratio in patients who underwent radical cystectomy for bladder cancer. Can Urol Assoc J 2015;9:e789-794.

-31 Mano R, Baniel J, Shoshany O, Margel D, Bar-On T, Nativ O, Rubinstein J, Halachmi S. Neutrophil-tolymphocyte ratio predicts progression and recurrence of non-muscle-invasive bladder cancer. Urol Oncol Semin Orig Investig 2015;33:67e1-67e7.

-32 Morizawa Y, Miyake M, Shimada K, Hori S, Tatsumi Y, Nakai Y, Anai S, Tanaka N, Konishi N, Fujimoto K. Neutrophil-to-lymphocyte ratio as a detection marker of tumor recurrence in patients with muscleinvasive bladder cancer after radical cystectomy. Urol Oncol Semin Orig Investig 2016;34:257.e11-257.e17.

33 Favilla V, Castelli T, Urzì D, Reale G, Privitera S, Salici A, Russo GI, Cimino S, Morgia G. Neutrophil to lymphocyte ratio, a biomarker in non-muscle invasive bladder cancer: A single-institutional longitudinal study. Int Braz J Urol 2016;42:685-693.

34 Mbeutcha A, Shariat SF, Rieken M, Rink M, Xylinas E, Seitz C, Lucca I, Mathieu R, Rouprêt M, Briganti A, Karakiewicz PI, Klatte T. Prognostic significance of markers of systemic inflammatory response in patients with non-muscle-invasive bladder cancer. Urol Oncol Semin Orig Investig 2016;34:483.e17-483.e24.

-35 Bhindi B, Hermanns T, Wei Y, Yu J, Richard PO, Wettstein MS, Templeton A, Li K, Sridhar SS, Jewett MAS, Fleshner NE, Zlotta AR, Kulkarni GS. Identification of the best complete blood count-based predictors for bladder cancer outcomes in patients undergoing radical cystectomy. Br J Cancer 2016;114:207-212.

-36 Kawahara T, Furuya K, Nakamura M, Sakamaki K, Osaka K, Ito H, Ito Y, Izumi K, Ohtake S, Miyoshi Y, Makiyama K, Nakaigawa N, Yamanaka T, , Miyamoto H, Yao M, Uemura H. Neutrophil-to-lymphocyte ratio is a prognostic marker in bladder cancer patients after radical cystectomy. BMC Cancer. BMC Cancer 2016;16:185.

-37 Buisan O, Orsola A, Oliveira M, Martinez R, Etxaniz O, Areal J, Ibarz L. Role of In $\mathrm{fl}$ ammation in the Perioperative Management of Urothelial Bladder Cancer With Squamous-Cell Features : Impact of Neutrophil-to-Lymphocyte Ratio on Outcomes and Response to Neoadjuvant Chemotherapy. Clin Genitourin Cancer 2017;15:e697-e706.

38 Hirasawa Y, Nakashima J, Yunaiyama D, Sugihara T, Gondo T, Nakagami Y, Horiguchi Y, Ohno Y, Namiki K, Ohori M, Tokuuye K, Tachibana M. Sarcopenia as a Novel Preoperative Prognostic Predictor for Survival in Patients with Bladder Cancer Undergoing Radical Cystectomy 2016;23:1048-1054.

39 Ogihara K, Kikuchi E, Yuge K, Yanai Y. The Preoperative Neutrophil-to-lymphocyte Ratio is a Novel Biomarker for Predicting Worse Clinical Outcomes in Non- muscle Invasive Bladder Cancer Patients with a Previous History of Smoking 2016;23:1039-1047.

-40 Kang M, Jeong CW, Kwak C, Kim HH, Ku JH. Preoperative neutrophil-lymphocyte ratio can significantly predict mortality outcomes in patients with non-muscle invasive bladder cancer undergoing transurethral resection of bladder tumor. Oncotarget 2017;8:12891-12901. 


\section{Cellular Physiology Cell Physiol Biochem 2018;46:1352-1364 and Biochemistry Published online: April 23, $2018 \quad \begin{aligned} & \text { DOI 101590018 The Author(s). Published by S. Karger AG, Basel } \\ & \text { www.karger.com/cpb }\end{aligned}$ \\ Wu et al.: NLR as a Predictor in BC, mUC or uUC}

41 D’Andrea D, Moschini M, Gust K, Abufaraj M, Ozsoy M, Mathieu R, Soria F, Briganti A, Roupret M, Karakiewicz PI, Shariat SF. Prognostic Role of Neutrophil-to-Lymphocyte Ratio in Primary Non-muscleinvasive Bladder Cancer. Clin Genitourin Cancer 2017;15:e755-e764.

42 Bambury RM, Benjamin DJ, Chaim JL, Zabor EC, Sullivan J, Garcia-Grossman IR, Regazzi AM, Ostrovnaya I, Apollo A, Xiao H, Voss MH, Iyer G, Bajorin DF, Rosenberg JE. The Safety and Efficacy of Single-Agent Pemetrexed in Platinum-Resistant Advanced Urothelial Carcinoma: A Large Single-Institution Experience. Oncologist 2015;20:508-515.

43 Taguchi S, Nakagawa T, Matsumoto A, Nagase Y, Kawai T, Tanaka Y, Yoshida K, Yamamoto S, Enomoto Y, Nose Y, Sato T, Ishikawa A, Uemura Y, Fujimura T, Fukuhara H, Kume H, Homma Y. Pretreatment neutrophil-tolymphocyte ratio as an independent predictor of survival in patients with metastatic urothelial carcinoma: A multi-institutional study. Int J Urol 2015;22:638-643.

$\checkmark 44$ Rossi L, Santoni M, Crabb SJ, Scarpi E, Burattini L, Chau C, Bianchi E, Savini A, Burgio SL, Conti A, Conteduca V, Cascinu S, De Giorgi U. High neutrophil-to-lymphocyte ratio persistent during first-line chemotherapy predicts poor clinical outcome in patients with advanced urothelial cancer. Ann Surg Oncol 2015;22:13771384.

45 Su Y, Hsieh M, Chiang P, Sung M, Lan J, Luo H. Novel Inflammation-Based Prognostic Score for Predicting Survival in Patients with Metastatic Urothelial Carcinoma 2017;12:1-12.

-46 Thompson DB, Siref LE, Feloney MP, Hauke RJ, Agrawal DK. Immunological basis in the pathogenesis and treatment of bladder cancer. Expert Rev Clin Immunol 2015;11:265-279.

47 Grivennikov SI, Greten FR, Karin M. Immunity, Inflammation, and Cancer. Cell 2011;140:883-899.

48 Jackaman C, Tomay F, Duong L, Abdol Razak NB, Pixley FJ, Metharom P, Nelson DJ. Aging and cancer: The role of macrophages and neutrophils. Ageing Res Rev 2017;36:105-116.

-49 Cao J, Zhu X, Zhao X, Li X-F, Xu R. Neutrophil-to-Lymphocyte Ratio Predicts PSA Response and Prognosis in Prostate Cancer: A Systematic Review and Meta-Analysis. PLoS One 2016;11:e0158770.

-50 Huang Y, Sun Y, Peng P, Zhu S, Sun W, Zhang P. Prognostic and clinicopathologic significance of neutrophilto-lymphocyte ratio in esophageal squamous cell carcinoma: Evidence from a meta-analysis. Onco Targets Ther 2017;10:1165-1172.

51 Kim HS, Ku JH. Systemic Inflammatory Response Based on Neutrophil-to-Lymphocyte Ratio as a Prognostic Marker in Bladder Cancer. Dis Markers 2016;2016:8345286.

52 Cao J, Zhao X, Zhong Z, Zhang L, Zhu X, Xu R. Prognostic Value of Pre-operative Renal Insufficiency in Urothelial Carcinoma: A Systematic Review and Meta-Analysis. Sci Rep 2016;6:1-9.

-53 Rose TL, Milowsky MI. Improving Systemic Chemotherapy for Bladder Cancer. Curr Oncol Rep. Current Oncology Reports 2016;18:27.

54 González del Alba A, Arranz JÁ, Puente J, Méndez-Vidal MJ, Gallardo E, Grande E, Pérez-Valderrama B, González-Billalabeitia E, Lázaro-Quintela M, Pinto Á, Lainez N, Piulats JM, Esteban E, Maroto Rey JP, García JA, Suárez C. Recent advances in genitourinary tumors: A review focused on biology and systemic treatment. Crit Rev Oncol Hematol 2017;113:171-190.

55 Viers BR, Boorjian SA, Frank I, Tarrell RF, Thapa P, Karnes RJ, Thompson RH, Tollefson MK. Pretreatment neutrophil-to-lymphocyte ratio is associated with advanced pathologic tumor stage and increased cancerspecific mortality among patients with urothelial carcinoma of the bladder undergoing radical cystectomy. Eur Urol. European Association of Urology 2014;66:1157-1164.

-56 Kessel KEM Van, Haan LM De, Fransen EE, Putte V De. Elevated Derived Neutrophil-to-Lymphocyte Ratio Corresponds With Poor Outcome in Patients Undergoing Pre-Operative Chemotherapy in Muscle-Invasive Bladder Cancer 2016;2:351-360.

57 Tang X, Du P, Yang Y. The clinical use of neutrophil-to-lymphocyte ratio in bladder cancer patients: a systematic review and meta-analysis. Int J Clin Oncol 2017;22:817-825.

58 Albayrak S, Zengin K, Tanik S, Atar M, Unal SH, Imamoglu MA, Gurdal M. Can the neutrophil-to-lymphocyte ratio be used to predict recurrence and progression of non-muscle-invasive bladder cancer? Kaohsiung J Med Sci 2016;32:327-333.

-59 Zhang G-M, Zhu Y, Luo L, Wan F-N, Zhu Y-P, Sun L-J, Ye D-W. Preoperative lymphocyte-monocyte and platelet-lymphocyte ratios as predictors of overall survival in patients with bladder cancer undergoing radical cystectomy. Tumour Biol 2015;36:8537-8543. 